\title{
Glycosaminoglycan therapy for long-term diabetic complications?
}

\author{
G. Gambaro ${ }^{1}$, J.Skrha ${ }^{2}$, A. Ceriello ${ }^{3}$ \\ ${ }^{1}$ Institute of Internal Medicine, Division of Nephrology, University of Padua, Italy \\ 2 3rd Department of Internal Medicine, Charles University, Prague, Czech Republic \\ ${ }^{3}$ Department of Internal Medicine, University of Udine, Italy
}

Long-term complications are the most important cause of mortality of diabetic patiens in western countries and diabetic nephropathy has emerged as a major determinant of end-stage renal failure [1]. Moreover, patients with diabetes mellitus have a high probability of developing acute cardiovascular disease, in particular myocardial infarction and cerebrovascular stroke which are the cause of death in nearly $80 \%$ of this population [2]. Although data from the Diabetes Control and Complications Trial establish that hyperglycaemia has a central role in diabetic complications, strict metabolic control can be difficult to achieve. The search for new and ancillary approaches to diabetic complications is therefore warranted and understanding the distal pathway of glucose toxicity assumes clinical and therapeutical significance. Thus, evidence has been provided $[3,4]$ that the alterations of the glycosaminoglycan (GAG) structure and metabolism may be of major importance for the development of diabetic complications. The aim of this article is to stress the hypothesis that GAGs might play an important role in the pathogenesis of late diabetic complications and that their treatment may be useful in several of these complications.

\section{GAG structure and function}

GAGs are highly glycosylated and sulphated glycoproteins, consisting of dimeric repeated units containing an uronic acid (iduronic or glucuronic acid)

Corresponding author: Dr A. Ceriello, Chair of Internal Medicine, University of Udine, P.le S.Maria della Misericordia, 33100 Udine, Italy

Abbreviations: GAGs, Glycosaminoglycans; GF, growth factors; HS, heparan sulphate; HS-PG, heparan sulphate-proteoglycan; t-PA, tissue plasminogen activator. and an aminosugar (glucosamine or galattosamine) [5]. These molecules are widely distributed in the body and prominent in extracellular matrices [5].

Three major classes of GAGs have been described: a predominant large chondroitin sulphate, a small dermatan sulphate and a polydisperse heparan sulphate (HS) [5]. GAGs are vital in maintaining the structural integrity of the tissue and studies have shown that basement membranes contain HS in the form of a proteoglycan unique to that tissue [5]. HS forms anionic sites in this matrix and are thought to restrict the passage of proteins through the basement membrane [5].

\section{GAG metabolism and diabetes mellitus}

In diabetic patients, the increased macromolecular permeability within the glomeruli that precedes the onset of established renal lesions and the increased vascular permeability to albumin seem to be related to structural alterations in the macromolecular pathway, i.e. the extracellular matrix, between the endothelial cells [4]. The nature of this alteration may be related to the loss of negatively charged molecules of extracellular matrix and of the endothelium, among which HS is highly represented. A number of reports indicate that HS metabolism is impaired in diabetes and this is responsible for the extracellular matrix negative charge loss [4]. HS is a strongly negatively charged molecule that is structurally similar to heparin but its base polymer has a lower degree of processing (sulphation, epimerization) (Fig. 1). The HS chain imparts a negative charge to the basement membrane and determines the molecular charge permselectivity of the glomerular basement membrane [4]. The reduction in HS negative charges may depend on either a decreased sulphation of the glycosaminoglycan molecule or an absolute reduction 


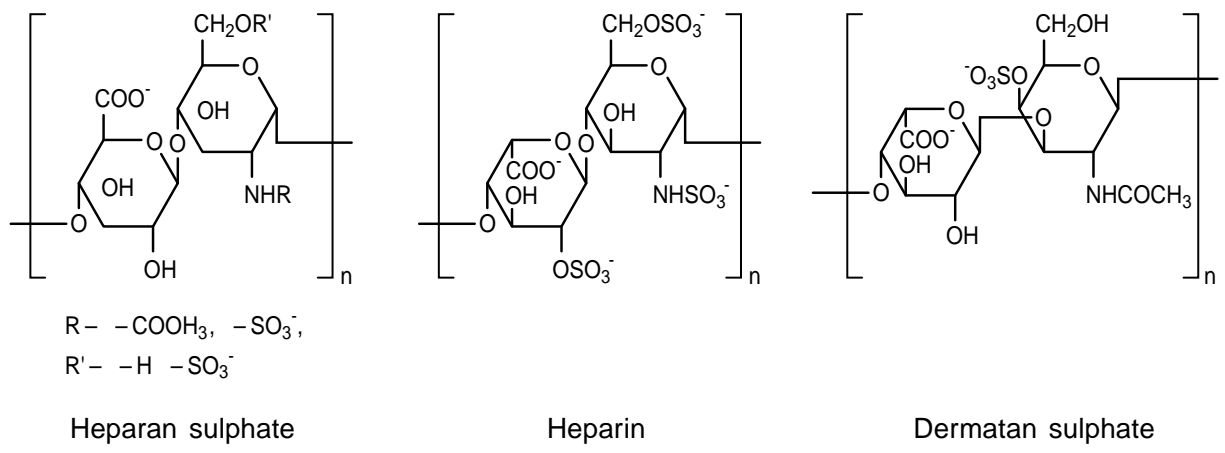

Fig. 1. Chemical structures of heparan sulphate, heparin and dermatan sulphate

in the heparan sulphate-proteoglycan (HS-PG). It has been suggested that the first HS-PG change in diabetes might be an undersulphation of HS, followed by an absolute, or most likely a relative decrease in HS [6].

\section{Abnormal GAGs and diabetic nephropathy}

The altered GAG metabolism in the diabetic kidney, and the abnormal GAG and extracellular matrix composition of the glomerular basement membrane may be important determinants of diabetic nephropathy, glomerulosclerosis, and renal failure due to the abnormal glomerular permselectivity to proteins and albuminuria. Data support the hypothesis that increased glomerular trafficking of proteins has detrimental effects on nephropathies, and in many primary glomerulopathies, proteinuria is certainly a risk factor for the development of glomerulosclerosis and renal failure [7]. However, the altered GAG or PG composition or both may be an important factor in the determination and the progression of diabetic nephropathy not only because of the abnormal glomerular basement membrane. The qualitative and quantitative changes in the PG composition of the renal extracellular matrix could also deeply affect the growth and synthetic behaviour of renal endothelial, mesangial, and glomerular and tubular epithelial cells, thus inducing glomerulosclerosis; indeed, the cell/extracellular matrix interaction plays an important role in regulating the adhesion, migration and proliferation of these cells [8]. Moreover, extracellular matrix and cell-associated PGs may modulate the activity of growth factors. In particular, it was suggested that the cell surface and extracellular matrix HS-PG may act together to regulate the bioavailability of otherwise diffusible effector molecules to their signal transducting receptor [8]. Note that a huge number of growth factors and cytokines are associated with HS-PGs and many are, or may be, involved in the pathogenesis of diabetic nephropathy [9]. Finally, it has recently been suggested that oxidative stress is involved in the pathogenesis of diabetic complications [10]. Further mesangial cells subjected to oxidative stress have been shown to selectively reduce HS synthesis [11].

\section{GAG treatment of diabetic nephropathy}

The treatment of diabetic nephropathy with GAGs was originally proposed on the basis of the somewhat simple idea that restoring the lacking anionic charges and GAGs to the diabetic kidney could cure the albuminuria and putatively return the above described cell functional anomalies to normal [12] (Fig. 2). The observation of pathophysiological similarities between diabetic nephropathy and the socalled "remnant kidney", i.e. reduced functioning tissue after a renal lesion [13], was also considered. In the classical model reproducing this kind of lesion, the 5/6 subtotal nephrectomized rat, heparin and derived drugs were shown to be effective in slowing down the progression to uraemia [14]. In view of these considerations, the effect of heparin and GAGs on diabetic nephropathy was studied in the streptozotocin diabetic model. This approach reduced glomerular basement membrane thickening [12] and anionic charge loss [12], as well as mesangial area expansion [15], and prevented the onset of albuminuria $[12,16]$ and the disorder in charge permselectivity [16]. Marshall et al. [15], however, were not able to confirm a reduction of albumin excretion in female streptozotocin diabetic Wistar rats on a twice daily dose of 200 units heparin over a period of 6 months. They did, though, report that basement membrane thickness, mesangial volume fraction and absolute mesangial volume were lower in heparintreated diabetic animals compared with untreated diabetic animals [15]. Recently these discrepancies have been discussed extensively [17]. The difference in the results may be mainly due to different qualities and quantities of drugs in the different protocols [17].

Heparin and related drugs have no effect on the metabolic control of diabetes. Since heparin and 

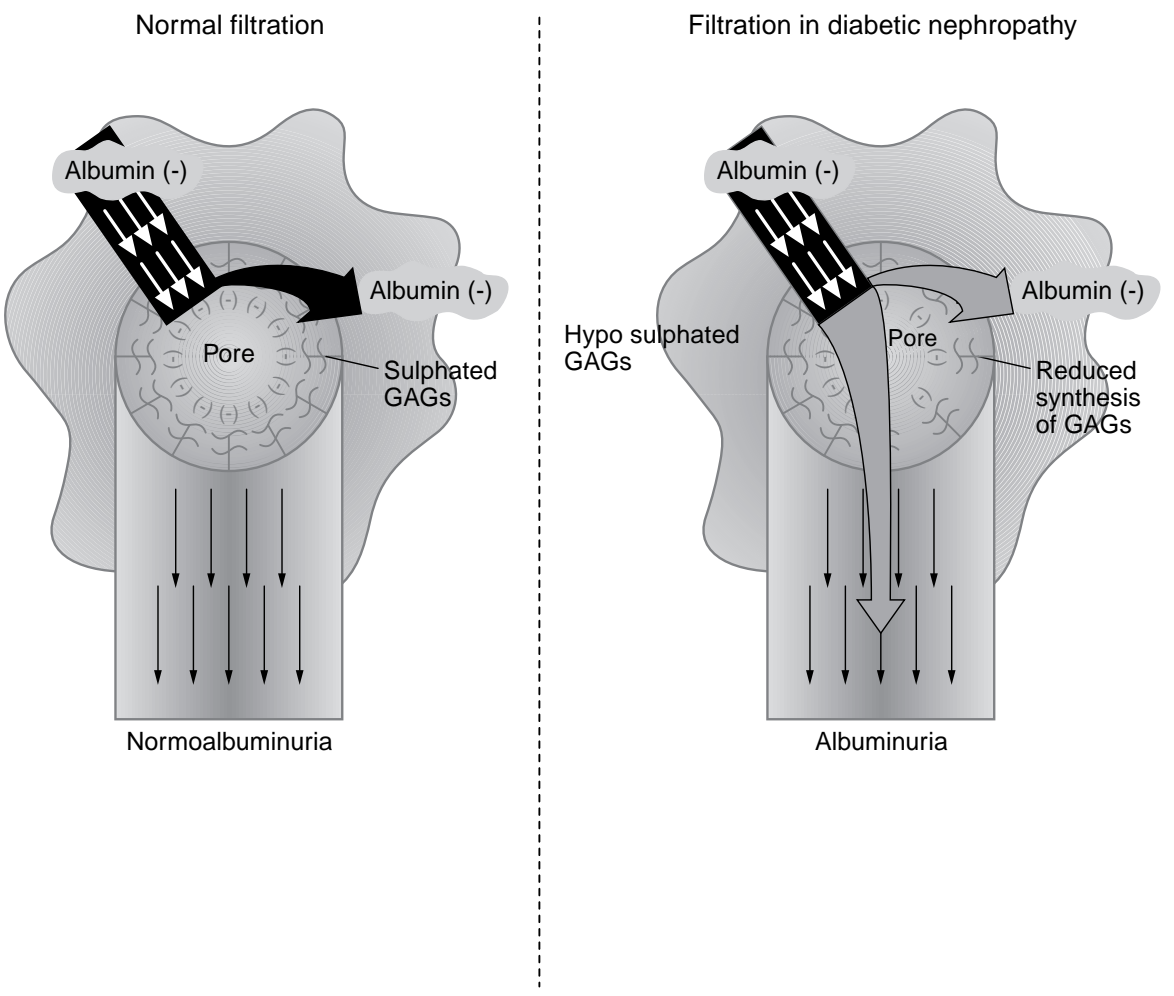

Filtration after GAG therapy
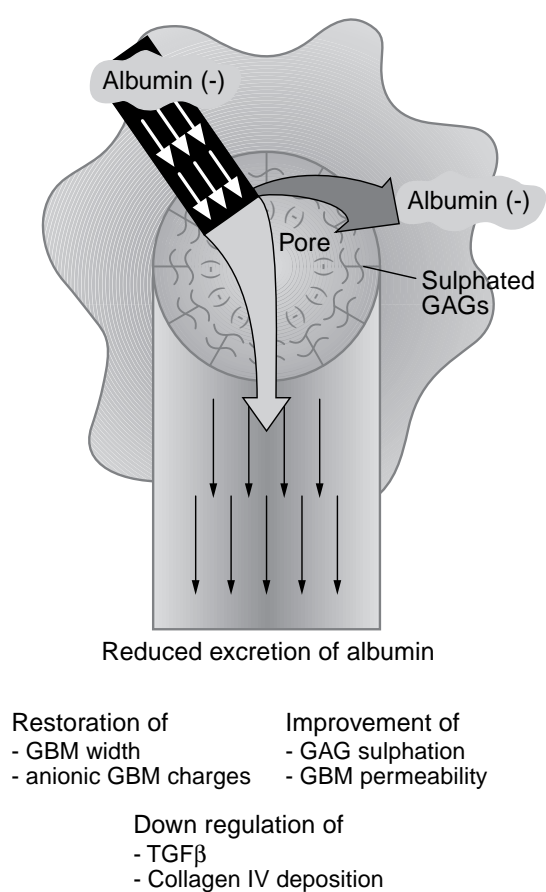

Fig. 2. Hypothetical mechanisms leading to albuminuria in diabetic patients and to curative effects of glycosaminoglycans. The glycosaminoglycan-dependent negative charge of the basement membrane determines the permselectivity of glomeruli to albumin. In diabetes a loss of glycosaminoglycans favours albumin excretion. The administration of glycosaminoglycans restores the negative charge of basement membrane, thus reducing albuminuria

GAGs have been demonstrated to modify the synthesis of PGs and other matrix proteins in vascular smooth muscle and endothelial cells by influencing extracellular matrix composition $[18,19]$ they could affect glomerular cell production of matrix/glomerular basement membrane constituents, and correct the diabetic extracellular matrix abnormalities responsible for glomerular basement membrane thickening and glomerulosclerosis. Indeed, in the streptozotocin model, heparin improved sulphate glomerular uptake, a metabolic marker of the endogenous GAG metabolism which is depressed in diabetes [16] and reduced collagen gene over-expression and the deposition of its protein [16]. In the streptozotocin model, heparin down-regulates collagen over-expression by interfering with the transforming growth factor (TGF)- $\beta 1$ autocrine/paracrine loop, which is known to be activated in the diabetic kidney and is thought to play a very important role in the pathogenesis of this complication [9]. Low molecular weight dermatan sulphate, the GAG derivative that prevents the onset of diabetic renal alterations in the streptozotocin model [12], also inhibits protein-ki-
nase-C-dependent activation of the TGF- $\beta 1$ gene in mesangial cells in vitro [20].

In the light of these findings, studies were conducted in diabetic patients. Recent reports described favourable results on proteinuria in diabetic nephropathy following treatment with different GAGs. Treatment with a low molecular weight heparin reduced albuminuria in both micro- and macroalbuminuric Type I diabetic patients [21-22]. Danaparoid, a mixture of sulphated GAGs composed mainly of HS, also lowered proteinuria in a small double-blind, crossover study in Type I diabetic patients with albumin excretion rates higher than $300 \mathrm{mg} / 24 \mathrm{~h} \mathrm{[23].} \mathrm{A} \mathrm{formulation} \mathrm{composed} \mathrm{of} \mathrm{the}$ two GAGs that were active in preventing diabetic nephropathy in the experimental model [12] was also tested in patients. Indeed, sulodexide, a GAG formulation composed of $80 \%$ fast-moving heparin and $20 \%$ dermatan sulphate, was reported to reduce albuminuria in Type I and Type II diabetic patients $[24,25]$, and this effect lasted several weeks after its withdrawal [26]. This decrease in albuminuria was also documented in a cross-over study [27] and in patients to whom sulodexide was given orally [24, 27].

\section{GAGs, microalbuminuria, diabetic nephropathy and cardiovascular risk}

As mentioned above, atherosclerosis is the main cause of death in diabetic patients and cardiovascular complications are frequently associated with diabetic 
nephropathy. It is noteable that the urinary excretion of albumin is significantly correlated with the incidence of mortality for cardiovascular events, in both Type I and Type II diabetic patients [3].

According to the "Steno Hypothesis", the increased risk of cardiovascular disease associated with nephropathy may be explained on the basis that HS metabolism in diabetes mellitus is not only altered within the glomerulus, but also within the capillary walls and the intima of large vessels [28]. Studies on muscle have demonstrated that the HS content of the basement membrane in muscle capillaries is reduced in diabetic patients with nephropathy and, noteably, is inversely correlated to the degree of albuminuria [29]. HS is also reduced in the intima of large vessels [30].

The altered HS-PG metabolism in the vessel intima and luminal endothelial membrane may be a consequence of endothelial dysfunction in microalbuminuric diabetic patients. Reduced HS synthesis by endothelial cells might cause thrombophilia in diabetes mellitus because, due to its negative charge, HS regulates the development of pericellular thrombotic phenomena at the level of the endothelial membrane, by interacting with antithrombin III [31]. This hypothesis is supported by studies which found an increased ratio between fibrinogen and antithrombin III, due to a decrease in antithrombin III, in the aorta of diabetic rats [32] and the observation of increased levels of markers of thrombin activation in diabetic patients [33]. As recently suggested, this last condition may produce an increase in fibrinogen [34], a well recognized cardiovascular risk factor also in diabetes [35]. The fall in endothelial cell HS contents might also influence local fibrinolysis. Endothelial cells synthesize specific HS and chondroitin sulphate to which tissue plasminogen activator (t-PA) can bind [36]. This t-PA binding capacity might be important in forming a t-PA storage pool, from which t-PA is released and made locally available under certain circumstances [36]. HSbound t-PA released from endothelial cells would provide an additional mechanism for locally increased fibrinolytic activity and render endothelial cells resistant to fibrin deposition. Thus, the derangement in this fibrinolytic mechanism could lead to increased fibrin deposition on the vascular wall in diabetes.

\section{Perspectives}

Although only small studies have been conducted, the favourable effects of GAG treatment on albuminuria in diabetic nephropathy are encouraging. Clearly, dose-finding and long-term studies are needed to demonstrate that these drugs are capable of curing human diabetic nephropathy and not simply albu- minuria. Interest, however, in GAGs is increasing, since these molecules have shown a beneficial effect on diabetic retinopathy [37]. Moreover, these drugs might retard the natural development of diabetic macroangiopathy and thrombosis. Indeed, the existence of common features (i.e. abnormal permeability to albumin) and pathogenetic mechanisms (i.e. altered HS metabolism, extracellular matrix deposition, overactivity of growth factors loops) between diabetic nephropathy and diabetic macroangiopathy and the ability of GAGs to act favourably on specific disorders of the vessel wall and endothelium (i.e. those disorders which lead to thrombophilia) suggests the possibility that these drugs might also be effective in preventing or curing or both not only diabetic nephropathy, but also diabetic macroangiopathy.

An increasing number of studies are showing that the administration of GAGs lowers fibrinogen levels, the relevance of which as a cardiovascular risk factor in diabetes has already been stressed [35]. In particular, the fibrinogen reducing activity of sulodexide was demonstrated in hyperlipidaemic subjects [38] and in pilot studies in diabetic patients [25, 39]. GAGs are also effective in stimulating fibrinolysis, and sulodexide was shown to improve fibrinolysis in diabetic patients [40]. Finally, heparin-like GAGs may modulate the procoagulant properties of endothelial cells through the increase of HS at the endothelial membrane level [19]. That these GAG activities may have therapeutic relevance in cardiovascular disease is supported by the reduction in total mortality, reinfarction rate and mural thrombus formation observed in patients treated with sulodexide after acute myocardial infarction [41].

Further studies of the structure-function relationship of heparin and GAGs and their optimal dosage may provide a unique opportunity to select new derivatives with specific effects on diabetic nephropathy and macroangiopathy, possibly and most importantly without anticoagulation. Such a prospect might be possible because some of the previously reported GAG effects depend on their non-sulphated molecular back-bone or are reached at non-anticoagulant dosages or both. Studies on the bioavailability of orally dispensed GAGs would also be very important. It has been demonstrated that after oral administration heparin and dermatan sulphate are absorbed [42-44] and, most importantly, maintain their effects not only on coagulation, but on albuminuria, as observed by Solini et al. [27] in diabetic patients treated with sulodexide. 


\section{References}

1. Striker GE, Agodoa LL, Held P, Doi T, Conti F, Striker LJ (1991) Kidney disease of diabetes mellitus (diabetic nephropathy): perspectives in the United States. J Diabetes Complications 5: 51-52

2. Colwell JA (1993) Vascular thrombosis in type II diabetes mellitus. Diabetes 42: 8-11

3. Deckert T, Kofoed-Enevoldsen A, Norgaard K, Borch-Johnsen K, Feldt-Rasmussen B, Jensen T (1992) Microalbuminuria. Implications for micro- and macrovascular disease. Diabetes Care 1992 15: $1181-11913$

4. Gambaro G, Baggio B (1992) Role of glycosaminoglycans in diabetic nephropathy. Acta Diabetol 29: 149-155

5. Muir H, Hardingham TE (1976) Structure of proteoglicans. In MTP Inter Rev Sci Biochemistry of carbohydrates. Biochemistry Series One Vol 5 (ed) Whelan Butterworths WJ, University Park Press

6. Born van den J, Berden JHM (1995) Is microalbuminuria in diabetes due to changes in glomerular heparan sulphate? Nephrol Dial Transplant 10: 1277-1296

7. Remuzzi G (1995) Abnormal protein traffic through the glomerular barrier induces proximal tubular cell dysfunction and causes renal injury. Curr Opin Nephrol Hypertens 4: 339-342

8. Ruoslahti E (1989) Proteoglycans in cell regulation. J Biol Chem 264: $13369-13372$

9. Gambaro G, Baggio B (1998) Growth factors and the kidney in diabetes. Crit Rev Clin Lab Sci 34: 1-24

10. Giugliano D, Ceriello A, Paolisso G (1996) Oxidative stress and diabetic vascular complications. Diabetes Care 19: 257-267

11. Kashihara N, Wanatabe Y, Makino H, Wellner EI, Kanwar YS (1992) Selective decreased de novo synthesis of glomerular proteoglycans under the influence of reactive oxygen species. Proc Natl Acad Sci USA 89: 6309-6313

12. Gambaro G, Cavazzana AO, Luzi P et al. (1992) Glycosaminoglycans prevent morphological renal alterations and albuminuria in diabetic rats. Kidney Int 42: 285-291

13. Hostetter TH, Rennke HG, Brenner BM (1982) The case for intrarenal hypertension in the initiation and progression of diabetic and other glomerulopathy. Am J Med Sci 72: 375-380

14. Purkerson ML, Tollefsen DM, Klahr S (1988) N-Desulfated/ Acetylated heparin ameliorates the progression of renal disease in rats with subtotal renal ablation. J Clin Invest 81: 69-74

15. Marshall SM, Hansen KW, Østerby R, Frystyk J, Ørskov H, Flyvbjerg A (1996) Effects of heparin on renal morphology and albuminuria in experimental diabetes. Am J Physiol 271 34: E326-E332

16. Gambaro G, Venturini AP, Noonan DM et al. (1994) Treatment with a glycosaminoglycan formulation ameliorates experimental diabetic nephropathy. Kidney Int 46: 797-806

17. Gambaro G, Baggio B (1998) Heparin and diabetic nephropathy. Am J Physiol 274: E192 Letter

18. Cochran DL, Castellot JJ, Karnovsky MJ (1985) Effect of heparin on vascular smooth muscle cells. II.Specific protein synthesis. J Cell Physiol 124: 29-36

19. Nader HB, Buonassisi V, Colburn P, Dietrich CP (1989) Heparin stimulates the synthesis and modifies the sulfation pattern of heparin sulfate proteoglycan from endothelial cells. J Cell Physiol 140: 305-310

20. Schleicher E, Ceol M, Sauer U, Baggio B, Gambaro G (1997) In: Pontuch P, Mojto V, Kozá Ková S (eds) Glycosaminoglycan treatment prevents induction of TGF-b expression and independently induces perlecan expression in mesangial cells. Proc 10th Meeting of the European Diabetic Nephropathy Study Group, Slovak Medical Association Bratislava, $\mathrm{p} 86$

21. Myrup B, Hansen PM, Jensen T et al. (1995) Effect of low-dose heparin on urinary albumin excretion in insulin-dependent diabetes mellitus. Lancet 345: 421-422

22. Tamsma JT, Woude van der FJ, Lemkes HHPJ (1996) Effect of sulfated glycosaminoglycans on albuminuria in patients with overt diabetic (type-1) nephropathy. Nephrol Dial Transplat 11:182-185

23. Pijl van der JW, Woude van der FJ, Geelhoed-Duijvestijn PHLM et al. (1997) Danaparoid sodium lowers proteinuria in diabetic nephropathy. J Am Soc Nephrol 8: 456-462
24. Solini A, Carraro A, Barzon I, Crepaldi G (1994) Therapy with glycosaminoglycans lowers albumin excretion rate in non-insulin dependent diabetic patients with microalbuminuria. Nutr Metab 7: 304-307

25. Velussi M, Cernigoi AM, Dapas F, De Monte A (1996) Glycosaminoglycans oral therapy reduces microalbuminuria, blood fibrinogen levels and limb arteriopathy clinical signs in patients with non-insulin dependent diabetes mellitus. Nutr Metab 9: $53-58$

26. Skrha J, Perusicová J, Pontúch P, Oksa A (1997) Glycosaminoglycan sulodexide decreases albuminuria in diabetic patients. Diabetes Res Clin Pract 38: 25-31

27. Solini A, Vergnani L, Ricci F, Crepaldi G (1997) Glycosaminoglycans delay the progression of nephropathy in NIDDM. Diabetes Care 20: 813-817

28. Deckert T, Feldt-Rasmussen B, Borch-Johnsen K, Jensen T, Kofoed-Enevoldsen A (1989) Albuminuria reflects widespread vascular damage: the Steno Hypothesis. Diabetologia 32: 219-226

29. Hansen PM, Høier PE, Yokoyama H et al. (1996) Loss of capillary basement membrane heparan sulphate in IDDM patients with nephropathy: a universal alteration? Diabetologia 39 [Suppl 1] A43 (Abstract)

30. Wasty F, Alavi MZ, Moore S (1993) Distribution of glycosaminoglycans in the intima of human aortas: changes in atherosclerosis and diabetes mellitus. Diabetologia 36: 316-322

31. Stern DM, Esposito C, Gerlach H et al. (1991) Endothelium and regulation of coagulation. Diabetes Care 14 [Suppl 1]:160-166

32. Witmer MR, Hadcock SJ, Peltier SL, Winocour PD, Richardson M, Hatton MW (1992) Altered levels of antithrombin III and fibrinogen in the aortic wall of the alloxan-induced diabetic rabbit: evidence of a prothrombotic state. J Lab Clin Med 119: 221-230

33. Ceriello A (1993) Coagulation activation in diabetes mellitus: the role of hyperglycaemia and therapeutic prospects. Diabetologia 36: $1119-1125$

34. Ceriello A, Taboga C, Giacomello R et al. (1994) Fibrinogen plasma levels as a marker of thrombin activation in diabetes. Diabetes 43: 430-432

35. Ceriello A (1997) Fibrinogen and diabetes mellitus: is it time for intervention trials? Diabetologia 40: 731-734

36. Böhm T, Geiger M, Binder BR (1996) Isolation and characterization of tissue-type plasminogen activator-binding proteoglycans from human umbilical vein endothelial cells. Arterioscler Thromb Vasc Biol 16: 665-672

37. Pijl van der JW, Woude van der FJ, Swart W, van Es LA, Lamkes NHPJ (1997) Effect of danaparoid sodium on hard exudates in diabetic retinopathy. Lancet 350: 1743-1745

38. Crepaldi G, Fellin R, Calabrò A et al. (1990) Double-blind multicenter trial on a new medium molecular weight glycosaminoglycan. Current therapeutic effects and perspectives for clinical use. Atherosclerosis 81: 233-243

39. Ceriello A, Quatraro A, Ettorre M, Marchi E, Barbanti M, Giugliano D (1993) Glucosaminoglycans administration decreases high fibrinogen plasma levels in diabetic patients. Nutr Metab 6: 203-206

40. Ceriello, Quatraro A, Marchi E, Barbanti M, Giugliano D (1993) Impaired fibrinolytic response to increased thrombin activation in type I diabetes mellitus: the effect of the glucosaminoglycan sulodexide. Diabetes Metab 19: 225-229

41. Condorelli M, Chiariello M, Daggianti A (1994) IPO-V2: a prospective, multicenter, randomized, comparative clinical investigation of the effects of Sulodexide in preventing cardiovascular accidents in the first year after acute myocardial infarction. J Am Coll Cardiol 23: 27-34

42. Jaques LB, Hiebert LM, Wice SM (1991) Evidence from endothelium of gastric absorption of heparin and of dextran sulfates 8000. J Lab Clin Med 117: 122-130

43. Hiebert LM, Wice SM, Jaques LB (1996) Antithrombotic activity of oral unfractionated heparin. J Cardiovasc Pharmacol 28: 26-29

44. Dawes J, Hodson BA, MacGregor IR, Pepper DS, Prowse CV (1989) Pharmacokinetic and biological activities of dermatan sulfate (Mediolanum MF701) in healthy human volunteers. Ann NY Acad Sci 556: 292-303 